\title{
A CASE OF LYMPHANGIOLEIOMYOMATOSIS WITH MULTIPLE ANGIOMYOLIPOMAS IN THE KIDNEYS: A CASE REPORT
}

\author{
How $\mathrm{SH}^{\prime}$, Azlin $\mathrm{S}^{2}$, Pang $\mathrm{YK}^{3}$ and Liam $\mathrm{CK}^{3}$ \\ Department of Internal Medicine, Kulliyyah of Medicine, International Islamic University Malaysia, P.O. Box 14 I, 257I0 Kuantan, Pahang \\ 2 Department of Radiology, Kulliyyah of Medicine, International Islamic University Malaysia \\ 3 Department of Medicine, Faculty of Medicine, University of Malaya, 50603 Kuala Lumpur, Malaysia
}

\begin{abstract}
Lymphangioleiomyomatosis (LAM) is a rare disease. The reported prevalence is around one per million in the West but the exact prevalence in Asia is unknown. It affects primarily young women of reproductive age, with a mean age of onset at 34 years. The disease is characterised by an abnormal proliferation of smooth muscle-like cells that grow over a course of time to obstruct airways, lymphatic and blood vessels. We report a case of LAM in a young woman who presented with a spontaneous pneumothorax. Computed tomography scan of the thorax and abdomen showed homogenous thin-walled cystic lesions with normal intervening lung parenchyma and multiple lesions in her kidneys with Hounsfield units similar to that of fat. (JUMMEC 2006; 9(2): 35-38)
\end{abstract}

KEYWORDS: Lymphangioleiomyomatosis, renal angiomyolipoma, spontaneous pneumothorax, cystic lesions of lungs

\section{Introduction}

Lymphangioleiomyomatosis (LAM) is a rare disease. The reported prevalence is around one per million population in the United Kingdom, France and the United States of America (I). The disease has been well documented in many Asian countries. However, the prevalence data of this disease in Asia is not available (I). It affects primarily young women of reproductive age, with a mean age of onset at 34 years. The disease is characterized by an abnormal proliferation of smooth muscle-like cells that grow over a course of time to obstruct airways, lymphatic and blood vessels. It usually occurs sporadically, but occasionally may occur in association with tuberous sclerosis complex. A case of LAM associated with renal angiomyolipoma is described with a particular focus on the diagnostic difficulty encountered.

\section{Case Report}

A 36 year old Chinese woman first presented in October 200I to the University of Malaya Medical Centre with a complaint of cough with episodes of haemoptysis for six months. She did not have reduced effort tolerance, fever, weight loss or reduced appetite. She denied taking any oral contraceptive pill. She used to smoke about ten cigarettes per day for three years during her teens. There was no history of tuberculosis infection or tuberculosis contact. She worked as an office clerk after completing her secondary school education.

Physical examination during the first hospital admission showed that she was comfortable at rest with no signs of respiratory distress. She did not have digital clubbing or fever. Findings on respiratory examination were unremarkable. Her chest radiograph was reported as "abnormalities consistent with bilateral generalised bronchiectasis". Her full blood and differential white cell counts, serum immunoglobulin and alpha-I-antitrypsin levels were normal.

Correspondence:

Dr Pang Yong Kek

Lecturer

Department of Medicine

Faculty of Medicine, University of Malaya

50603 Kuala Lumpur, Malaysia

Email:ykpang@ummc.edu.my 
Conventional spiral computed tomography (CT) scan of her thorax and abdomen performed after hospital discharge revealed "multiple cysts of varying sizes in the lungs, as well as multiple bilateral hypodense lesions in the kidneys with a Hounsfield unit of fat. Mild ascites and abdominal lymphadenopathy were also noted (Figure I and 2). These CT scan findings were thought to be consistent with LAM and multiple renal angiomyolipoma, probably secondary to underlying tuberous sclerosis. However, the patient was treated as having bronchiectasis despite the CT scan findings.

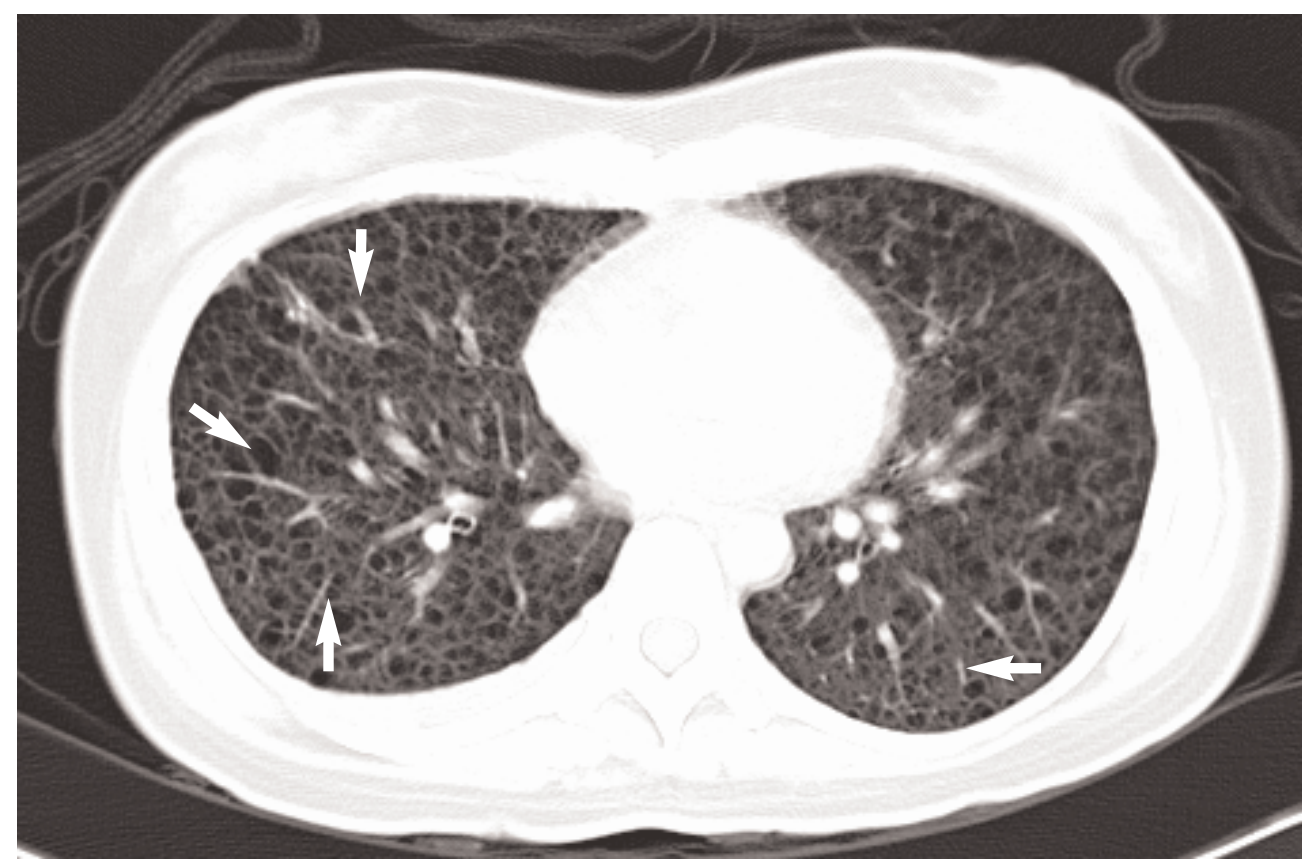

Figure I. White arrows show multiple thin-wall cystic lesions, which are quite homogenous in size scattered throughout both lungs. The intervening lung parenchyma appears normal.

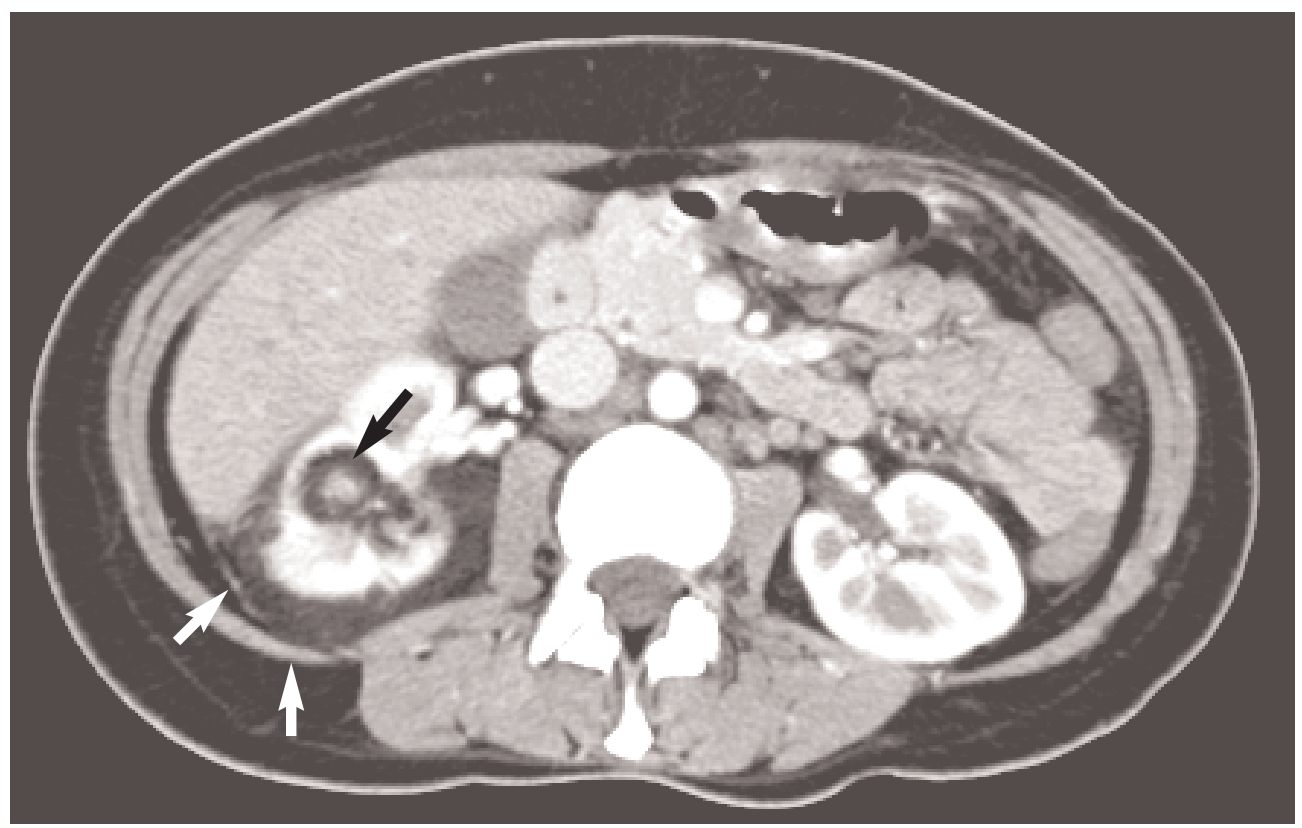

Figure 2. Contrasted axial-CT at the level of both kidneys revealed a lobulated mass in the right kidney (shown with black arrow) with density similar to that of fat (margins shown by white arrows). This finding is typical of renal angiomyolipoma. 
Sixteen months later in February 2003, she presented with a right-sided hydro-pneumothorax. Her chest radiograph also showed reticulonodular infiltrates and small cystic lesions in both lungs with blurring of the cardiac margins. The left lung volume was preserved. The right hydro-pneumothorax was drained by an intercostal chest tube under water-seal. Minimal straw-coloured fluid was drained. She was discharged two weeks later when the right lung was fully reexpanded.

On clinic follow-up, she remained quite well except for occasional episodes of haemoptysis. A review of the patient's thoracic CT scan which had been taken in 200I, revealed that she had had diffuse cystic lesions in the lungs without a predilection for central or peripheral involvement. These cystic lesions were thinwalled, quite homogenous in size and not adjacent to the vasculature of lung. The intervening lung parenchyma was normal. The clinical diagnosis was revised in February 2004 from bronchiectasis to that of LAM. A respiratory function test performed revealed a forced expiratory volume in the first second $\left(\mathrm{FEV}_{1}\right)$ of $58 \%$ of predicted; forced vital capacity (FVC), $78 \%$ of predicted; total lung capacity (TLC), $96 \%$ of predicted; residual volume (RV), $130 \%$ of predicted and an $\mathrm{FEV}_{1} /$ FVC ratio of $62 \%$. Her lung diffusing capacity (DLCO) was only $54 \%$ of predicted. She underwent an open lung biopsy of her right upper, middle and lower lobes which revealed emphysematous changes without typical changes of LAM on histopathological examination. As she only had minimal symptoms, she was not keen to undergo another lung biopsy and treatment. A repeat CT examination in January 2005 did not show any change in the lung lesions nor the size of the renal angiomyolipoma.

\section{Discussion}

Despite being described in scientific literatures since 1937, the aetiology of LAM remains elusive. However, hormonal factors particularly, oestrogen and progestorone seem to play a major role in the initiation, progression and retardation of the disease. This is based on the observations that the disease occurs exclusively in women, worsens during pregnancy and exacerbates after hormonal therapy in post-menopausal women (I). Treatment with progesterone and/or oophorectomy in certain reported series seems to afford retardation of disease progression or even improvement of the condition (I). Furthermore, oestrogen and progesterone receptors have been found in LAM tissue.
In a study in the United Kingdom (UK) by Johnson (2), the commonest presentations of LAM are pneumothorax (38.7\%), dyspnoea (36.7\%) followed by chylous pleural effusion (6.1\%). Renal angiomyolipoma was found in $12.0 \%$ of the patients in this series. If the incidence in Malaysia with a population of about 25 million is comparable to that of UK, we would expect to see around 25 cases of LAM in the country and we expect only two or three patients to have renal angiomyolipoma. Pulmonary function test may show an obstructive (commonest), restrictive or mixed pattern with reduced DLCO. As in this patient, the residual volume is usually increased (3).

The diagnosis of LAM can be difficult. In one series (3), the exact diagnosis was made after an average delay of 44 months (ranging from I to 219 months after the first symptoms). In our patient, the definite diagnosis was made after 36 months from the time of first presentation even though the diagnosis was suspected by a radiologist on the first presentation. This was probably due to the lack of awareness among physicians on this rare condition. Plain chest radiograph during the early stages of the disease is often normal in appearance. Later, when the disease advances, mild reticulonodular shadows or small cystic lesions would appear (I). These changes, however, are often misinterpreted as some other more common diseases, e.g., bronchiectasis, as seen in this patient. Hence, when clinical features of the disease are not compatible with radiological diagnosis based on chest radiograph, further investigation with high resolution CT scan is advisable.

The classical findings of LAM described on chest radiograph are bilateral reticulonodular interstitial infiltrates, with normal or increased lung volumes (3). However, these findings also occur in other diseases, such as chronic hypersensitivity pneumonitis, pulmonary histiocytosis $\mathrm{X}$, sarcoidosis and emphysema coexisting with idiopathic pulmonary fibrosis (3). High resolution thorax CT scan findings (as described above for this patient) are characteristic but not pathognomonic of LAM because patients with chronic histiocytosis $X$ may have similar CT appearances (2). Open lung biopsy has been the gold standard to diagnose this disease. However, in the presence of typical clinical features and characteristic $\mathrm{CT}$ findings of LAM, open lung biopsy may be omitted (I). Furthermore, abdominal CT scan in this patient also revealed features consistent with renal angiomyolipoma, ascites (which could have been chylous) and abdominal lymphadenopathy, which are well known association with LAM. 


\section{Conclusion}

In conclusion, the diagnosis of LAM should be considered when a young female presents with spontaneous pneumothorax and CT scan findings of homogenous thin-wall cystic lesions and normal intervening lung parenchyma.

\section{References}

I. Johnson S. Lymphangioleiomyomatosis: clinical features, management and basic mechanisms. Thorax 1999; 54: 254-264.

2. Johnson R, Tattersfield AE. Clinical experience of lymphangioleiomyomatosis in the UK. Thorax 2000; 55: 10521057.

3. Sullivan EJ. Lymphangioleiomyomatosis: a review. Chest 1998; I|4: I689-| 703. 